\title{
BMJ Open Determinants of frequent and infrequent STI testing and STI diagnosis related to test frequency among men who have sex with men in the eastern part of the Netherlands: a 6-year retrospective study
}

Carolina J G Kampman, ${ }^{1}$ Janneke C M Heijne, ${ }^{2}$ Petronella H M Kistemaker-Koedijk, ${ }^{1}$ Femke D H Koedijk, ${ }^{1}$ Maartje Visser, ${ }^{2}$ Jeannine L A Hautvast ${ }^{3}$

To cite: Kampman CJG, Heijne JCM, KistemakerKoedijk PHM, et al. Determinants of frequent and infrequent STI testing and STI diagnosis related to test frequency among men who have sex with men in the eastern part of the Netherlands: a 6-year retrospective study. BMJ Open 2018;8:e020495. doi:10.1136/ bmjopen-2017-020495

- Prepublication history for this paper is available online To view these files, please visit the journal online (http://dx.doi. org/10.1136/bmjopen-2017020495).

Received 7 November 2017 Revised 1 March 2018 Accepted 20 April 2018

Check for updates

${ }^{1}$ Public Health Service Twente, Enschede, The Netherlands

${ }^{2}$ Centre for Infectious Disease Control, National Institute for Public Health and the Environment, Bilthoven, The Netherlands

${ }^{3}$ Department of Primary and Community Care, Radboud University Medical Center, Nijmegen, The Netherlands

Correspondence to Drs. Carolina J G Kampman; k.kampman@ggdtwente.nl

\section{ABSTRACT}

Objective Men who have sex with men (MSM) remain vulnerable to sexually transmitted infections (STIS) and are advised to be tested at least twice a year. The aim of this study was to assess the determinants of test frequency and their associations with an STI diagnosis.

Design A 6-year retrospective study.

Setting 5 STI clinics in the eastern part of the Netherlands.

Participants MSM whose mean test interval was 6 months or more were grouped as 'infrequently tested' $(n=953)$, and those with a mean test interval less than 6 months were grouped as 'frequently tested' $(n=658)$.

Primary and secondary outcome measures Test frequency and STI diagnosis and determinants.

Results MSM who were ever diagnosed with an STI ( $\mathrm{OR}=1.4,95 \% \mathrm{Cl} 1.1$ to 1.7$)$, MSM who had never had STI symptoms ( $\mathrm{OR}=0.8,95 \% \mathrm{Cl} 0.6$ to 1.0$)$, and MSM who had ever had sex with both men and women $(\mathrm{OR}=0.6,95 \% \mathrm{Cl}$ 0.5 to 0.8 ) were more often frequently tested. Moreover, in both groups, MSM who had ever been notified by a partner $(\mathrm{OR}=2.2,95 \% \mathrm{Cl} 1.7$ to 2.9 infrequently tested; $\mathrm{OR}=2.0$, $95 \% \mathrm{Cl} 1.4$ to 2.9 frequently tested), MSM who had ever had STI symptoms $(\mathrm{OR}=1.6,95 \% \mathrm{Cl} 1.2$ to 2.1 infrequently tested; $\mathrm{OR}=1.8,95 \% \mathrm{Cl} 1.3$ to 2.6 frequently tested) and MSM who were ever diagnosed with HIV $(\mathrm{OR}=2.7,95 \% \mathrm{Cl}$ 1.5 to 4.6 infrequently tested; $\mathrm{OR}=6.8,95 \% \mathrm{Cl} 2.6$ to 17.5 frequently tested) were more likely to be diagnosed with an STI.

Conclusions Among MSM visiting STI clinics, those who were ever diagnosed with HIV were more often diagnosed with an STI, but did not visit STI clinics more frequently than HIV-negative MSM. This highlights the necessity of encouraging MSM who are diagnosed with HIV to have STI tests more frequently.

\section{INTRODUCTION}

Men who have sex with men (MSM) contribute to the worldwide burden of

\section{Strengths and limitations of this study}

- Longitudinal data of men who have sex with men (MSM) with at least three consultations at regional Dutch sexually transmitted infection (STI) clinics between 2008 and 2013 were analysed.

- The current study has a long follow-up of 5 years, so a large number of MSM were included in analyses.

- The MSM population in the eastern part of the Netherlands and their test behaviour may differ from the rest of the country.

- STI diagnosis was only available for those who came back for repeat testing; they do not represent the reinfections contracted by all MSM who visit STI clinics.

- Only STI clinic consultations were available; data from STI tests carried out by general practitioners or other specialists could not be taken into account.

sexually transmitted infections (STIs), including HIV. ${ }^{1-3}$ Some studies have found that MSM reported large proportions of diagnoses of HIV (42\%), gonorrhoea (43\%) and syphilis $(58 \%) .^{45}$ In the Netherlands, MSM accounted for the greatest contribution to STI diagnoses in 2016 at STI clinics; $21 \%$ of the tests were positive for one or more STIs. ${ }^{6}$

Early detection and treatment are crucial to reduce the risk of STI transmissions (including HIV) among MSM. ${ }^{7}$ Therefore, test frequency is important in reducing STI transmissions. In Australia, MSM are advised to get a test at least once a year, but one study reports that this advice is poorly adhered to: the retesting rate at 1 year was $35 \%{ }^{8}$ A British study estimates that $55 \%$ of the MSM were tested once a year, where 
guidelines also recommend HIV tests once a year (and more often for those at 'higher risk'). ${ }^{9}$

There were only informal guidelines for test frequency among MSM in the Netherlands before 2017, based on expert opinion. These informal guidelines recommended STI testing for MSM at least twice a year. A formal guideline, based on Dutch epidemiological findings, has now (2017) been drafted, and it advises MSM to be tested at least twice a year, and high-risk MSM (eg, MSM who were diagnosed with HIV or MSM who are commercial sex workers) four times a year. However, the annual testing uptake among MSM in the Netherlands is low. One Dutch study on STI consultations in Amsterdam from 2009 to 2013 reports that $35 \%$ of the HIV-negative MSM returned to the STI clinic within 1 year after their initial consultation. ${ }^{10}$ Another Dutch study based on national STI clinic data from 2014 to 2015 reports that $48 \%$ of the MSM were tested more than once during a 1.5-year follow-up. Only $19 \%$ of the MSM were tested consistently every 6 months. ${ }^{11}$

No studies have yet simultaneously investigated the relation between MSM test frequency and STI diagnosis, with the determinants, in the Netherlands. The aim of this study was to assess the determinants of test frequency and of STI diagnosis among MSM visiting STI clinics in the eastern part of the Netherlands. The results of the study could provide more insight into the frequency and relevance of testing according to the guidelines for certain MSM risk groups.

\section{METHODS}

\section{Study setting and design}

In the Netherlands, general practitioners and STI clinics based in regional public health services (RPHSs) provide primary STI care. The STI clinics are freely accessible and government-funded; they aim to reach high-risk groups who might otherwise not seek timely STI care. The STI clinic at the RPHS is always accessible to MSM, whether or not reporting STI-related symptoms, while there is a triage system for heterosexuals. Furthermore, MSM are always tested for five STIs: chlamydia, gonorrhoea, syphilis, HIV (unless clients opt out) and hepatitis B (when not successfully vaccinated against hepatitis B) ${ }^{6}$

We performed a 6-year retrospective study (2008-2013) using data from 5 of the 25 Dutch STI clinics. The participating clinics were in the east of the Netherlands, which is a semirural area. All of them used an online patient registration system in which sexual preference was a mandatory question. Data from 2013 onwards were not included due to changes in the patient registration system of the STI clinics. The definitions of database variables could not be matched.

\section{Study population}

We selected all men who reported having sex with men or sex with both women and men, and men who identified themselves as homosexual or bisexual (hereafter referred to as MSM). Only MSM who had three or more consultations were included in the study because three or more consultations were considered to approximate an actual test frequency. We selected MSM who had a minimum of 18 months of follow-up after the first consultation (which took place before June 2012, with a follow-up time extending into 2013) because all MSM had to have sufficient time to return for two retests. Furthermore, consultations within 35 days of a previous visit were excluded to ensure that no possible test-of-cure consultations were included.

\section{Data description}

For each consultation, the following variables were used: age $(<26$ years and $\geq 26$ years-clients younger than 26 years are considered 'young' as decided by the national STI clinic regulations); ethnicity (due to a change in registration, ethnicity was a combination of variables that consisted of self-defined ethnicity (from 2006 until 2010 ) and ethnicity based on (parental) country of birth (from 2011 until 2013); MSM were subsequently categorised into Dutch, other Western and non-Western); having been notified by a partner; having STI symptoms; socioeconomic status (SES, based on postal codes (four digits)) - this measure of SES was deduced by postal code-associated data from the Netherlands Institute for Social Research and is a measure composed of four variables: average income per household, percentage of households with low income, percentage of residents without a paid job and percentage of households with an average to low education; number of sexual partners in the last 6 months; being diagnosed with an STI during the study period; and sexual preference (reported as having sex with men or sex with men and women). HIV status was considered a separate variable based on existing literature that indicates that MSM who are diagnosed with HIV are more likely to be diagnosed with an STI. ${ }^{12-15}$ MSM were defined as diagnosed with HIV when they were diagnosed with HIV during the study period or were already diagnosed with HIV before the study period.

\section{Data analysis}

The determinants age, ethnicity and SES were taken from the first consultation in all analyses because these determinants are reasonably stable over time. The mean number of partners reported per consultation was used to determine the number of partners in the analysis. All other determinants were assessed on the basis of the occurrence of the event within all individual consultations, which resulted in an 'ever' and 'never' occurring categorisation.

The outcome test frequency was defined as follows: MSM were defined infrequently tested if their mean test intervals were 6 months or more. They were defined as frequently tested if their mean test intervals were less than 6 months. A 1-month margin was taken into account to ensure that a person would not be regarded 
as infrequently tested if the mean test interval was only slightly more than 6 months.

The outcome STI diagnosis was defined as follows: any one of the MSM was defined as being diagnosed with one or more STIs, including chlamydia, gonorrhoea, syphilis and/or infectious hepatitis B, in one or more body locations (oral, genital or anal) at one or more consultations during the study period.

If more than $5 \%$ of the values were missing for a variable, these missing values were included in the analysis in a separate category to reduce loss of data. We performed logistic regression analyses to identify determinants of testing frequency and STI diagnosis. Collinearity between variables was checked beforehand. We used the Enter method with multivariable logistic regression to further analyse determinants with a $p$ value less than 0.20 in univariable analyses. The multivariable logistic regression was corrected for the number of consultations because the reporting of an event (eg, ever having STI symptoms) is more likely when MSM visit the STI clinic more often. In all analyses, determinants with $\mathrm{p}<0.05$ were considered statistically significant. We present ORs and $95 \%$ CIs to show the associations between the determinants and the outcomes in tables 1 and 2. We used IBM SPSS V.22 software for the analyses.

\section{Patient and public involvement}

The study used routinely collected anonymous surveillance data. Hence, STI clinic visitors were not directly involved in the development or execution of this study, and neither could the results be disseminated to them.

\section{RESULTS}

\section{Study population and test frequency}

A total of 5954 MSM visited one of the five participating STI clinics between 2008 and 2013. A total of 1913 MSM had three or more consultations, of whom 1611 also had a minimal follow-up time of 18 months after the first consultation and thus were included. The group infrequently tested' consisted of 953 MSM (59.2\%), and the group 'frequently tested' consisted of 658 MSM (40.8\%).

Table 1 shows that among the infrequently tested MSM, $47.0 \%$ were ever diagnosed with an STI, compared with $64.6 \%$ of the frequently tested MSM. Table 1 also compares the characteristics of the two groups. Multivariable analysis showed that the frequently tested had more often been diagnosed with an STI (OR 1.4, 95\% CI 1.1 to 1.7$)$, were less likely to ever have reported STI-related symptoms (OR 0.8, 95\% CI 0.6 to 1.0 ) and had less often ever had sex with men only (OR $0.6,95 \%$ CI 0.5 to 0.8 ) than the infrequently tested.

\section{STI diagnosis}

Table 2 shows the determinants of having an STI diagnosis. The same determinants of STI diagnosis were identified in both groups: MSM who had ever been notified by a partner, MSM who had ever had STI-related symptoms and MSM who were ever diagnosed with HIV were more likely to have an STI diagnosis. In addition, frequently tested MSM who only had sex with men were more likely to have an STI diagnosis, which was not seen among infrequently tested MSM.

\section{DISCUSSION}

In this study, we found that $59.2 \%$ of the included MSM were infrequently tested (mean test interval $\geq 6$ months) and $40.8 \%$ were frequently tested (mean test interval $<6$ months). MSM who were ever diagnosed with an STI, MSM who had never had STI symptoms, and MSM who had ever had sex with men as well as women were more often frequently tested. Moreover, we found that the determinants for STI diagnosis were the same in both groups. MSM who had ever been notified by a partner, MSM who had ever had STI symptoms and MSM who were ever diagnosed with HIV were more likely to be diagnosed with an STI.

This is the first study in the Netherlands that addresses both test frequency and STI diagnosis among MSM who were and were not diagnosed with HIV. Furthermore, our study has a long follow-up of 5 years, so a large number of MSM were included in analyses. However, the study has several limitations. First, it took place in the eastern part of the Netherlands, which is a semirural area. The study population and their test behaviour may differ from the rest of the Netherlands. Second, STI diagnosis was only available for those who came back for repeat testing; they do not represent the reinfections contracted by all MSM who visit STI clinics. Third, in this study, only STI clinic consultations were available, so data from STI tests carried out by general practitioners or other specialists could not be taken into account. The number of STI consultations per individual might therefore be an underestimation, and MSM could have been categorised differently if consultations from other caregivers have been included. Fourth, due to changes in the patient registration system, we could not include data beyond the year 2013. The STI clinic has, however, always been freely accessible to MSM over the years. We do not think there have been any sudden changes in risk behaviour and/or test frequency among MSM; therefore, we think the data of the current study are still of importance to STI care nowadays. Fifth, we excluded MSM with only one consultation. We reasoned that leaving them out would provide us with a more valid overview of test frequency in those who appear to be a regular client of STI clinics.

With the study methods we chose, this study shows that, of all the MSM with at least three consultations, $41 \%$ were frequently tested and they had a mean test interval of less than 6 months. The methods of the current study differ widely from other comparable Dutch studies so comparison is difficult. ${ }^{10}{ }^{11}$ Vriend and colleagues ${ }^{, 10}$ study found that $16 \%$ of HIV-negative MSM returned for repeat tests within 6 months. We only included MSM with at least two subsequent tests and a minimum of 18 
Table 1 Characteristics of the frequently and infrequently tested groups and the determinants of test frequency for MSM who visited an STI clinic in the eastern part of the Netherlands, 2008-2013

\begin{tabular}{|c|c|c|c|c|}
\hline & \multirow[b]{2}{*}{$\begin{array}{l}\text { Infrequently tested } \\
(\mathrm{n}=953) \\
\mathrm{n}(\%)\end{array}$} & \multirow[b]{2}{*}{$\begin{array}{l}\text { Frequently tested } \\
(\mathrm{n}=658) \\
\mathrm{n}(\%)\end{array}$} & \multicolumn{2}{|c|}{ Frequently versus infrequently testec } \\
\hline & & & $\begin{array}{l}\text { Univariable analyses } \\
\text { OR }(95 \% \mathrm{Cl})\end{array}$ & $\begin{array}{l}\text { Multivariable } \\
\text { analysis } \\
\text { OR }(95 \% \mathrm{Cl})\end{array}$ \\
\hline Median number of consultations (IQR) & $4(3-5)$ & $6(4-9)$ & & \\
\hline \multicolumn{5}{|l|}{ Age (in years at baseline) } \\
\hline$<26$ & $242(25.4)$ & $140(21.3)$ & Ref & Ref \\
\hline$\geq 26$ & $711(74.6)$ & $518(78.7)$ & $1.3(1.0$ to 1.6$)$ & 0.9 (0.7 to 1.2$)$ \\
\hline \multicolumn{5}{|l|}{ Ethnicity (baseline) } \\
\hline Dutch & $857(89.9)$ & $584(88.8)$ & Ref & NT \\
\hline Non-Western & $62(6.5)$ & $47(7.1)$ & $1.1(0.8$ to 1.6$)$ & \\
\hline Other Western & $34(3.6)$ & $27(4.1)$ & $1.2(0.7$ to 2.0$)$ & \\
\hline \multicolumn{5}{|l|}{ Socioeconomic status (baseline) } \\
\hline Low & $281(29.5)$ & $183(27.8)$ & 1.0 (0.7 to 1.2$)$ & NT \\
\hline Intermediate & $349(36.6)$ & 256 (38.9) & 1.1 (0.9 to 1.4$)$ & \\
\hline High & $300(31.5)$ & 203 (30.9) & Ref & \\
\hline Missing & $23(2.4)$ & $16(2.4)$ & & \\
\hline \multicolumn{5}{|l|}{ Mean number of partners } \\
\hline$<2$ & $122(12.9)$ & $67(10.3)$ & Ref & Ref \\
\hline $2-5$ & $406(43.0)$ & 227 (35.0) & $1.0(0.7$ to 1.4$)$ & $1.0(0.7$ to 1.5$)$ \\
\hline$\geq 5$ & $416(44.1)$ & $354(54.6)$ & 1.6 (1.1 to 2.2$)$ & 1.2 (0.8 to 1.8$)$ \\
\hline Missing & $9(0.0)$ & $10(0.0)$ & & \\
\hline \multicolumn{5}{|l|}{ Notified by a partner } \\
\hline Never & $511(53.6)$ & 267 (40.6) & Ref & Ref \\
\hline Ever & $442(46.4)$ & $391(59.4)$ & 1.7 (1.4 to 2.1 ) & 1.1 (0.9 to 1.4$)$ \\
\hline \multicolumn{5}{|l|}{ Diagnosed with an STI } \\
\hline Never & $505(53.0)$ & $233(35.4)$ & Ref & Ref \\
\hline Ever & $448(47.0)$ & $425(64.6)$ & 2.1 (1.7 to 2.6 ) & 1.4 (1.1 to 1.7$)$ \\
\hline \multicolumn{5}{|l|}{ STI-related symptoms } \\
\hline Never & $454(47.6)$ & $273(41.5)$ & Ref & Ref \\
\hline Ever & $499(52.4)$ & $385(58.5)$ & $1.3(1.1$ to 1.6$)$ & 0.8 (0.6 to 1.0$)$ \\
\hline \multicolumn{5}{|l|}{ Sexual preference } \\
\hline Sex with men and women & $232(24.3)$ & 212 (32.2) & Ref & Ref \\
\hline Sex with men only & $714(74.9)$ & $442(67.2)$ & 0.7 (0.5 to 0.8$)$ & 0.6 (0.5 to 0.8$)$ \\
\hline Unknown & $7(0.7)$ & $4(0.6)$ & & \\
\hline \multicolumn{5}{|l|}{ Diagnosed with HIV } \\
\hline Never & $860(90.2)$ & $568(86.3)$ & Ref & Ref \\
\hline Ever & $93(9.8)$ & $90(13.7)$ & $1.5(1.1$ to 2.0$)$ & $0.8(0.5$ to 1.2$)$ \\
\hline
\end{tabular}

Values in bold were significant $(p<0.05)$; due to rounding into 1 decimal, 1.0 is not always significant.

Regression analysis corrected for the number of consultations.

MSM, men who have sex with men; NT, not tested in multivariable model, since $p>0.20$ in univariate analysis; ref, reference; STI, sexually transmitted infection.

months of follow-up, whereas Vriend and colleagues ${ }^{10}$ also included MSM only tested once in their analyses, which made the comparison of the proportions of the frequently tested group in their study and our study difficult. However, Vriend and colleagues also looked at a consistent 12-month testing among people with at least 3 years of follow-up (ie, three or more tests) and found an uptake of $36 \%$, which is more in line with our study.

Our results show that MSM who had ever been diagnosed with an STI are more often frequently tested. 
Table 2 Determinants of STI diagnosis in infrequently and frequently tested MSM who visited an STI clinic in the eastern part of the Netherlands, 2008-2013

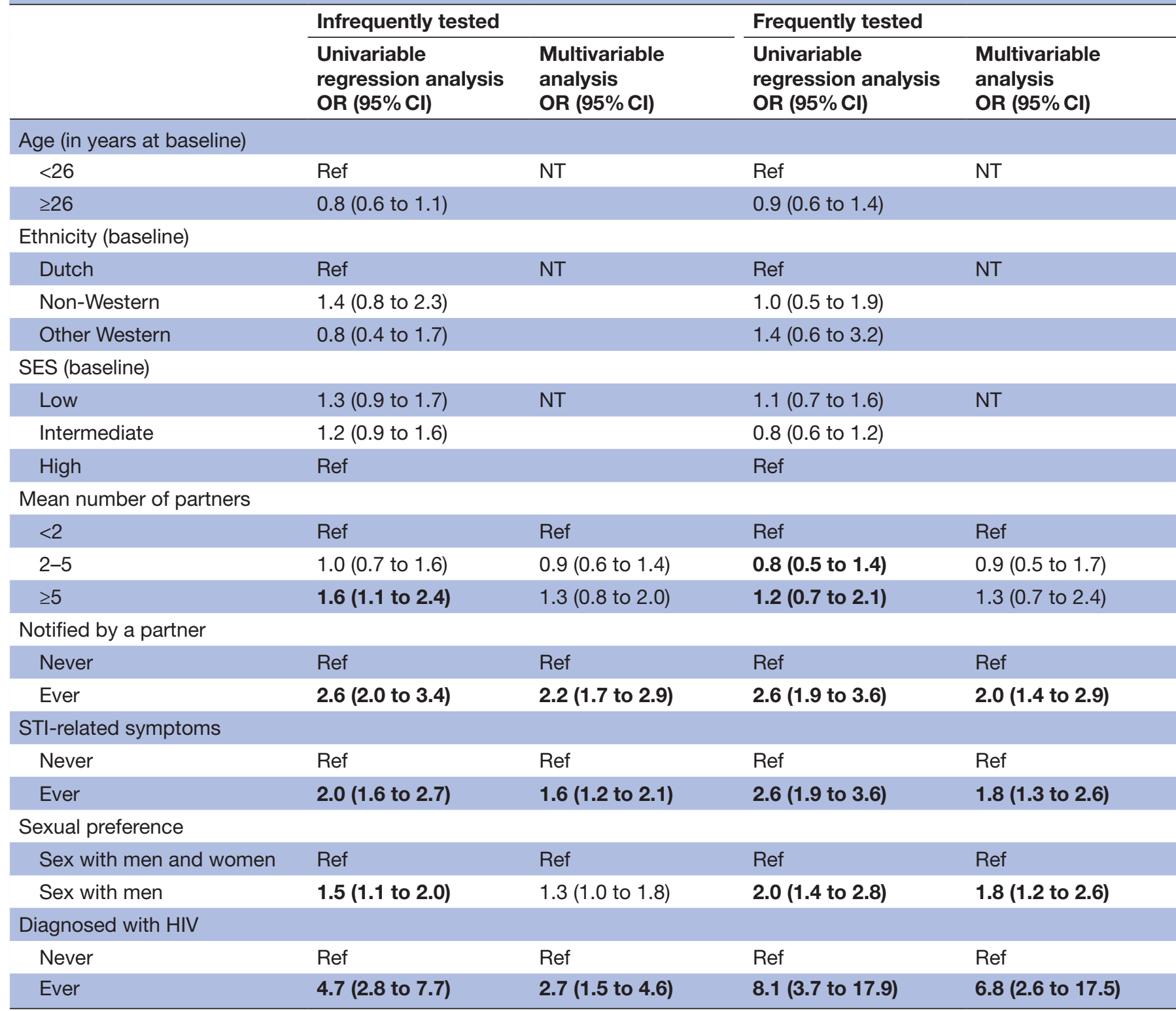

Values in bold were significant $(p<0.05)$; due to rounding into 1 decimal, 1.0 is not always significant.

Regression analysis corrected for the number of consultations.

MSM, men who have sex with men; ref, reference; SES, socioeconomic status; STI, sexually transmitted infection; NT, not tested in multivariate model, since $p>0.20$ in univariate analysis.

However, MSM who had ever been notified by a partner and MSM who had ever had STI-related symptoms are not more likely to be frequently tested. This is in line with another study which finds that MSM who have been notified by a partner or who have reported STI symptoms return to the STI clinic sooner, but are not more likely to be consistently tested every 6 months. ${ }^{11}$ Furthermore, our study shows that MSM who had ever had sex with men as well as women were more often frequently tested. In two other Dutch studies, men who had sex with both genders less often had repeat tests. ${ }^{10}{ }^{11}$ We do not have a clear explanation for this discrepancy, but a reporting bias in sexual preference could be a possible explanation. Further research is needed to gain more insight into this.
Regarding STI diagnosis, we show that MSM who had ever been notified by a partner, MSM who had ever had STI symptoms and MSM who were ever diagnosed with HIV were more likely to have an STI diagnosis. These results are in line with other studies. ${ }^{12-16}$ A British analysis using multiple sources of national surveillance data and population survey data concludes that an increasing proportion of STIs are being diagnosed in MSM who are diagnosed with HIV, with the population rate of STIs rising to four times that of HIV-negative or undiagnosed MSM. Moreover, STI reinfection rates were considerably higher in MSM who were diagnosed with HIV over a 5-year follow-up period. The authors believe the higher number of bacterial reinfections in MSM who are diagnosed with 
HIV are indicative of rapid transmission in dense sexual networks. ${ }^{17}$ An Italian study assessed risk behaviours before and after being diagnosed with HIV; MSM who are diagnosed with HIV continue to engage in at-risk practices: a quarter of them did not use condom during STI episodes, $12.5 \%$ of the participants had engaged in sex for money and $8.4 \%$ had paid for sex. ${ }^{18}$ Also, serosorting (selecting sex partners of the same HIV status) or assumed serosorting among MSM who are diagnosed with HIV may play a role in at-risk practices. Among MSM who are diagnosed with HIV, the likelihood of unprotected anal intercourse (UAI) is higher when a partner's status was known. Furthermore, assumed seroconcordant UAI is associated with increased STI prevalence. ${ }^{19-21}$

By combining the results of the significant determinants of frequent testing and STI diagnosis, this study demonstrates that MSM who had ever been notified by a partner and MSM who had ever had STI-related symptoms were more likely to have an STI diagnosis, but were not more likely to be frequently tested. This means that MSM who had symptoms or who had been notified by a partner appear to find their way to the STI clinics when necessary, but will not come back frequently. There is no legislation on partner notification in the Netherlands. Also, partner notification is performed anonymously; the STI clinic does not know to the full extent who is being notified.

We also found that MSM who were ever diagnosed with HIV were more often diagnosed with an STI, but did not visit the STI clinic more frequently than MSM who tested HIV-negative. Other studies also show that MSM who are diagnosed with HIV are more likely have an STI diagnosis. ${ }^{12-15}$ Routine screening for STI of MSM who were diagnosed with HIV is important because regular screening could help reduce the incidence of STI diagnoses. $^{22-24}$ MSM diagnosed with HIV are not routinely tested for STI in most HIV care centres, except for annual syphilis and hepatitis C screening. Dutch STI clinics put great efforts in motivating MSM to test for STIs by outreach activities at MSM events and providing anonymous online test facilities. This study highlights the importance of ongoing efforts done by STI clinics in encouraging MSM who were diagnosed with HIV to be tested for STI frequently.

Acknowledgements The authors thank all the medical staff of the five participating STI clinics for allowing us to use their data. We would also like to thank Birgit van Benthem and Koos van der Velden for their constructive comments on the manuscript.

Contributors CJGK contributed to statistical analysis, drafted the article and processed comments made by the other authors. JCMH discussed the methodological issues, gave advice about the statistics and made corrections to the article. PHMK-K drafted the research protocol, collected the data and assisted with the literature search. FDHK contributed to statistical analysis and commented on the article. MV discussed the methodological issues, gave advice about statistics and made corrections to the article. JLAH commented on the research protocol, helped with the data analysis and commented on the article. All authors gave their final approval of the final version of the original article to be published.

Funding The authors have not declared a specific grant for this research from any funding agency in the public, commercial or not-for-profit sectors.
Competing interests None declared.

Patient consent Not required.

Ethics approval Ethical approval for the study was not necessary in Dutch law because the study used routinely collected surveillance data that were anonymous.

Provenance and peer review Not commissioned; externally peer reviewed.

Data sharing statement There are no additional unpublished data from the study available.

Open Access This is an Open Access article distributed in accordance with the Creative Commons Attribution Non Commercial (CC BY-NC 4.0) license, which permits others to distribute, remix, adapt, build upon this work non-commercially, and license their derivative works on different terms, provided the original work is properly cited and the use is non-commercial. See: http://creativecommons.org/ licenses/by-nc/4.0/

(C) Article author(s) (or their employer(s) unless otherwise stated in the text of the article) 2018. All rights reserved. No commercial use is permitted unless otherwise expressly granted.

\section{REFERENCES}

1. Sullivan PS, Hamouda O, Delpech V, et al. Reemergence of the HIV epidemic among men who have sex with men in North America, Western Europe, and Australia, 1996-2005. Ann Epidemiol 2009;19:423-31.

2. European Centre for Disease prevention and Control. STI and HIV prevention in men who have sex with men in Europe. Stockholm: ECDC, 2013.

3. Cohen J, Lo YR, Caceres CF, et al. WHO guidelines for HIV/ STI prevention and care among MSM and transgender people: implications for policy and practice. Sex Transm Infect 2013;89:536.

4. European Centre for Disease prevention and Control. Surveillance report HIVIAIDS surveillance in Europe 2013. Stockholm: ECDC, 2014.

5. European Centre for Disease prevention and Control. Surveillance report Sexually transmitted infections in Europe 2013. Stockholm: ECDC, 2015

6. Visser M, van Aar F, van Oeffelen AAM. Sexually transmitted infections including HIV, in the Netherlands in 2016. RIVM 2017.

7. Center for Disease Control and Prevention. Sexually transmitted diseases treatment advises. MMWR 2010;59.

8. Guy R, Goller JL, Spelman T, et al. Does the frequency of HIV and STI testing among men who have sex with men in primary care adhere with Australian guidelines? Sex Transm Infect 2010;86:371-6.

9. McDaid LM, Aghaizu A, Frankis J, et al. Frequency of HIV testing among gay and bisexual men in the UK: implications for HIV prevention. HIV Med 2016;17:683.

10. Vriend HJ, Stolte IG, Heijne JC, et al. Repeated STI and HIV testing among HIV-negative men who have sex with men attending a large STI clinic in Amsterdam: a longitudinal study. Sex Transm Infect 2015;91:294.

11. Visser M, Heijne JCM, Hogewoning AA, et al. Frequency and determinants of consistent STI/HIV testing among men who have sex with men testing at STI outpatient clinics in the Netherlands: a longitudinal study. Sex Transm Infect 2017;93:396-403.

12. de Coul EL, Warning TD, Koedijk FD. Sexual behaviour and sexually transmitted infections in sexually transmitted infection clinic attendees in the Netherlands, 2007-2011. Int J STD AIDS 2014;25:40-51.

13. Koedijk FDH, van Benthem BHB, Vrolings EMDC, et al. Increasing sexually transmitted infection rates in young men having sex with men in the Netherlands, 2006-2012. Emerg Themes Epidemiol 2014;11:12.

14. Schmidt AJ, Hickson F, Weatherburn P, et al. Comparison of the performance of STI screening services for gay and bisexual men across 40 European cities: results from the European MSM internet Survey. Sex Transm Infect 2013;89:575.

15. Mirandola M, Gios L, Joanna Davis R, et al. Socio-demographic factors predicting HIV test seeking behaviour among MSM in 6 EU cities. Eur J Public Health 2017;27:313-8.

16. Wilkinson A, El-Hayek C, Fairley CK, et al. Incidence and risk factors associated with chlamydia in men who have sex with men: a cohort analysis of Victorian Primary Care Network for Sentinel Surveillance data. Sex Transm Infect 2012;88:319-24.

17. Malek R, Mitchell $\mathrm{H}$, Furegato $\mathrm{M}$, et al. Contribution of transmission in HIV-positive men who have sex with men to evolving epidemics of 
sexually transmitted infections in England: an analysis using multiple data sources, 2009-2013. Euro Surveill 2015;20:20.

18. Camoni L, Dal Conte I, Regine V, et al. Sexual behaviour reported by a sample of Italian MSM before and after HIV diagnosis. Ann Ist Super Sanita 2011;47:214-9.

19. Matser A, Heijman T, Geskus R, et al. Perceived HIV status is a key determinant of unprotected anal intercourse within partnerships of men who have sex with men in Amsterdam. AIDS Behav 2014;18:2442-56.

20. Hotton AL, Gratzer B, Mehta SD. Association between serosorting and bacterial sexually transmitted infection among HIV-negative men who have sex with men at an urban lesbian, gay, bisexual, and transgender health center. Sex Transm Dis 2012;39:959-64.
21. Zablotska IB, Imrie J, Prestage G, et al. Gay men's current practice of HIV seroconcordant unprotected anal intercourse: serosorting or seroguessing? AIDS Care 2009;21:501-10.

22. Dougan S, Evans BG, Elford J. Sexually transmitted infections in Western Europe among HIV-positive men who have sex with men. Sex Transm Dis 2007;34:783.

23. Xiridou M, Vriend HJ, Lugner AK, et al. Modelling the impact of chlamydia screening on the transmission of HIV among men who have sex with men. BMC Infect Dis 2013;13:436.

24. Vriend HJ, Lugnér AK, Xiridou $M$, et al. Sexually transmitted infections screening at HIV treatment centers for MSM can be costeffective. AIDS 2013;27:2281-90. 